\title{
A History of Recurrent Episodes of Prolonged Cough as a Predictive Value for Determining Cough Variant Asthma in a Primary Care Setting
}

\author{
Masaaki Mikami,* Katsuyuki Tomita $\uparrow$ and Akira Yamasaki: \\ *Hosshoji Internal Clinic, Nanbu 683-0351, Japan, †Department of Respirology Medicine, National Hospital Organization Yonago \\ Medical Center, Yonago 683-8518, Japan, and $¥$ Division of Medical Oncology and Molecular Respirology, School of Medicine, Faculty \\ of Medicine, Tottori University, Yonago 683-8503, Japan
}

\section{ABSTRACT}

Background Many patients visit primary care clinics with a complaint of cough. General practitioners (GPs) developed a list of the causative diseases of cough that can produce a patient's symptoms and signs. Then, the patients' medical histories were evaluated to determine whether the diagnosis of cough variant asthma (CVA) or post-infectious cough (PIC) could have been predicted.

Methods We retrospectively investigated 195 outpatients with a complaint of cough. Medical histories of "recurrent episodes of prolonged cough" and "upper respiratory infection" were obtained during the initial visit. The accuracy of medical histories in predicting CVA and PIC was calculated on the area under the curve (AUC).

Results Among eligible patients with cough, PIC was diagnosed in 99 patients $(50.8 \%)$, CVA in 40 patients (20.5\%), upper airway cough syndrome in 28 patients (14.4\%), and chronic obstructive pulmonary disease in 11 patients (5.6\%). Among the patients with CVA and those with PIC, $93 \%$ and $12 \%$, respectively, had a history of recurrent episodes of prolonged cough. For the diagnosis of CVA, having a history of recurrent episodes of prolonged cough showed a moderately accurate AUC (0.76, 95\% CI: 0.71-0.82). On the other hand, for the diagnosis of PIC, having no history of recurrent episodes of prolonged cough also showed a moderately accurate AUC (0.87, 95\% CI: 0.82-0.92).

Conclusion The medical history of recurrent episodes of prolonged cough is useful for the prediction of CVA as well as PIC.

Key words cough variant asthma; post-infectious cough; prolonged cough; recurrent episodes

A cough is the most common complaint when patients visit their primary care physician, accounting for around $8 \%$ of all consultations. ${ }^{1,2}$

In most cases, it is difficult to diagnose the cause of the cough. The first step in making a differential diagnosis of the cough is to confirm the duration of the cough. ${ }^{3-6}$ Generally, the classification of cough based on duration is as follows: acute cough, within 3 weeks of onset; sub-acute cough, from 3 to 8 weeks; and chronic cough, more than 8 weeks. This classification system is a useful tool for narrowing down the potential causes of cough. ${ }^{3-6}$

In adults, causes of acute cough include acute upper respiratory infection, acute rhinosinusitis, and allergic rhinitis. ${ }^{7}$ Post-infectious cough (PIC), which is defined as a self-limiting cough that persists after viral or virus-like infections, is the most common etiology of subacute cough. ${ }^{8,9}$ Approximately $12 \%-48 \%$ of adult patients with subacute cough have PIC.8, 10

Chronic cough is caused by cough variant asthma (CVA), upper airway cough syndrome (UACS), and gastro-esophageal reflux disease (GERD). ${ }^{11}$ In Japan, CVA is common among diseases that cause of subacute and chronic cough. ${ }^{12}$ The only symptom of CVA is cough with no wheezing or dyspnea, which is defined as a subtype of asthma that manifests only as a cough. In CVA, respiratory function tests are almost normal; airway hyperreactivity is mildly increased; and bronchodilator ( $\beta 2$-agonist) is effective. ${ }^{6,13}$ In adults, CVA evolves into typical asthma in $30 \%-40 \%$ of cases, but the rate of transition to typical asthma is reduced by using inhaled steroids at the time of early diagnosis. ${ }^{14,15}$ Therefore, accurate diagnosis and early therapeutic intervention are important for improving treatment efficacy and the resolution of symptoms of cough.

General practitioners can generally diagnose patients accurately based on their pattern of symptoms, response to medications, and family history. There is recent evidence that clinicians can make an accurate diagnosis of the cause of cough for most patients by

Corresponding author: Masaaki Mikami, MD, PhD

mmhsje@gmail.com

Received 2021 October 1

Accepted 2021 November 5

Online published 2021 November 29

Abbreviations: AC, atopic cough; ACE, angiotensin-converting enzyme inhibitor; AUC, area under the curve; $\mathrm{CB} / \mathrm{COPD}$, chronic bronchitis/chronic obstructive pulmonary disease; CVA, cough variant asthma; GERD, gastro-esophageal reflex disease; GPs, general practitioners; OSAS, obstructive sleep apnea syndrome; PIC, post-infectious cough; ROC, receiver operating characteristic; UACS, upper airway cough syndrome 
using their medical history alone. ${ }^{16}$ Tomita and colleagues reported that patients with a high probability of asthma were those with nocturnal cough/wheezing and/ or recurrent episodes of cough/wheezing. ${ }^{17}$ The medical history often provides important initial clues about patients with subacute or chronic cough. According to a diagnostic algorithm for the approach to adults with cough, all patients should be questioned about the use of angiotensin-converting enzyme (ACE) inhibitors and the presence of an upper respiratory tract infection at the onset of coughing. ${ }^{18}$ The aim of this study was to investigate the utility of typical histories of "recurrent episodes of prolonged cough" and "upper respiratory infection" as diagnostic indicators of causes of cough in a primary care setting.

\section{MATERIALS AND METHODS Patient population}

The subjects were 230 consecutive patients (aged 18-91 years) with a chief complaint of cough who were referred to our clinic as outpatients between January 2012 and December 2019. We first performed a plain chest X-ray test, blood test, spirometry, sputum eosinophilia, and chest and sinus computerized tomography as needed. Respiratory infections, such as pneumonia, tuberculosis, and nontuberculous mycobacteriosis, and other pulmonary diseases, including lung cancer, metastatic lung tumor, rheumatic lung, malignant lymphoma, and heart failure, were excluded from this study. Cases of bronchial asthma with wheezing were also excluded from this study.

Medical records of patients who met the inclusion criteria were investigated. These records included the medical history, smoking history, presence or absence of upper respiratory tract infection, atopic diseases, recurrent episodes of prolonged cough, total duration of cough, characteristics of cough and sputum, postnasal drip, heartburn, medication with an ACE inhibitor, previous visits to other clinics, and previous medical treatment. Regarding the history of prolonged cough, we asked questions such as, "Have you ever had a prolonged cough similar to this episode?"

This study was conducted in accordance with the Declaration of Helsinki and with the approval of the Tottori Prefecture Medical Association Ethics Committee (accession number: 2020-01). Written informed consent was obtained from all subjects.

\section{Definition of acute/subacute/chronic cough}

Current cough was divided into subtypes. Acute cough was defined as cough lasting less than 3 weeks; subacute cough was defined as cough lasting between 3 and 8 weeks; and chronic cough was defined as cough lasting for more than 8 weeks.

\section{Diagnosis of causative diseases}

In our approach to diagnosing causative diseases, we followed the American College of Chest Physicians Guidelines for the management of cough. ${ }^{19}$

CVA was diagnosed in patients who experienced a cough without wheezing for more than 3 weeks but did not have wheezing on auscultation. A sputum eosinophil test, respiratory tract reversibility test, and fractional exhaled nitric oxide measurement, which was measured using NIOX VERO ${ }^{\circledR}$ (Aerocrine, Sweden) with a cutoff of $30 \mathrm{ppb},{ }^{20}$ were evaluated as additional diagnostic tools. Based on these examinations, patients with suspected CVA were treated with long-acting $\beta 2$-agonist for 1-2 weeks and were observed for signs of improved cough. If improvement was observed, a treatment with an inhaled corticosteroid/long-acting $\beta 2$-agonist (ICS/ LABA) combination was started. Diagnosis of CVA was confirmed after the patient presented with a complete release of cough.

PIC was identified as a continuous cough after experiencing an upper respiratory tract infection, and a final diagnosis was performed if the administration of cough suppressants, histamine $\mathrm{H} 1$ antagonist, and/or Chinese herbal medicine ${ }^{21}$ effectively improved cough at a follow-up of 1-2 weeks.

Upper airway cough syndrome (UACS) was defined as cough with nasal blockage or nasal discharge (anterior or posterior nasal drip) and either facial pain/ pressure or reduction/loss of smell. ${ }^{22}$

Esophageal reflux disease was identified if there was heartburn and/or regurgitation at least one day per week for the past 3 months. ${ }^{23}$

Atopic cough was diagnosed following the diagnostic criteria for general clinical settings: (1) dry cough for at least 3 weeks without wheezing or dyspnea, (2) no response to bronchodilator therapy, (3) one or more findings suggesting an atopic predisposition or induced sputum eosinophilia, and (4) relief of cough with histamine $\mathrm{H} 1$ antagonists and/or steroids. ${ }^{24}$ Atopy was also defined as a self-reported allergy to pollens, animals, or food.

To reach an accurate diagnosis in situations where an empirical treatment of PIC or UACS did not improve symptoms and CVA was strongly suspected, an inhaled corticosteroid (ICS) treatment was performed.

Only cases for which there was a total resolution of cough after a follow-up period of 3 months or more were examined in this study. We thoroughly inquired about the symptoms by phone or other means for cases 
Table 1. Diagnosis classified by the duration of cough $(n=195)$

\begin{tabular}{lcccc}
\hline Diagnosis & Total $(n=195)$ & Acute $(n=29)$ & Subacute $(n=114)$ & Chronic $(n=52)$ \\
& $n(\%)$ & $n(\%)$ & $n(\%)$ & $n(\%)$ \\
\hline PIC & $99(50.8)$ & $22(75.9)$ & $72(63.2)$ & $5(9.6)$ \\
CVA & $40(20.5)$ & $0(0.0)$ & $27(23.7)$ & $13(25.0)$ \\
UACS & $28(14.4)$ & $6(20.7)$ & $9(7.9)$ & $13(25.0)$ \\
CB/COPD & $11(5.6)$ & $1(3.0)$ & $2(1.8)$ & $8(15.4)$ \\
AC & $7(3.6)$ & $0(0.0)$ & $3(2.6)$ & $4(7.7)$ \\
GERD & $6(3.1)$ & $0(0.0)$ & $0(0.0)$ & $6(11.5)$ \\
OSAS & $2(1.0)$ & $0(0.0)$ & $0(0.0)$ & $2(3.8)$ \\
ACE-induced & $2(1.0)$ & $0(0.0)$ & $1(0.9)$ & $1(1.9)$ \\
\hline
\end{tabular}

$\mathrm{AC}$, Atopic cough; ACE, Angiotensin-converting enzyme inhibitor; CB/COPD, Chronic bronchitis/Chronic obstructive pulmonary disease; CVA, Cough variant asthma; GERD, Gastro-esophageal reflex disease; OSAS, Obstructive sleep apnea syndrome; PIC, Postinfectious cough; UACS, Upper airway cough syndrome.

that did not return to the clinic for a 3-month followup. After confirmation that the cough had resolved, the etiologic treatment was judged to be effective, so a final diagnosis was made.

\section{Statistical analysis}

One-way analysis of variance (ANOVA) was used to determine whether there were any statistically significant differences in the continuous data among groups. A post-hoc analysis was performed using Scheffe's test. Differences in categorical variables between clusters were statistically assessed using Fisher's exact test, and the Benjamini-Hockberg procedure was performed to correct for multiple comparisons. ${ }^{25}$

Next, the accuracy of medical histories in predicting PIC and CVA was calculated in terms of sensitivity and specificity. Positive likelihood hood ratios (LR+) and negative likelihood ratios (LR-) were the primary measures of diagnostic accuracy. An LR+ greater than 10 (an estimated shift in probability of at least $45 \%$ ) is strong evidence to rule in a disease, whereas an LR+ between 5 and 10 is moderate (estimated shift of at least $30 \%$ ) and between 2 and 5 is weak (estimated shift $15 \%) .{ }^{26,27}$ A plot of specific receiver operating characteristic (ROC) curves was used as a graphic presentation of the relationship between both sensitivity and specificity; it helped to decide the optimal model. An area under the curve (AUC) of more than 0.9 was defined as high accuracy, between 0.9 and 0.7 as moderate accuracy, and less than 0.7 as low accuracy. ${ }^{28}$

For all statistical tests, the significance level was set at 0.05 . Analyses were conducted using R statistical software (version 3.5.2; R Foundation for Statistical Computing Vienna, Austria).

\section{RESULTS}

Among the 230 patients with cough who visited our clinic between January 2012 and December 2019, 16 presented with cases of organic diseases ( 8 cases of pneumonia, 2 of nontuberculous mycobacteriosis, 1 of lung cancer, 1 of metastatic lung tumor, 1 of sequela of pulmonary tuberculosis, 1 of rheumatic lung, 1 of malignant lymphoma, and 1 of heart failure); 2 presented with bronchial asthma; and 17 were undiagnosed cases, which were subsequently excluded from this study. Among these were 82 males and 113 females with an average age of $49.7 \pm 18.2$ years old; $21.5 \%$ were smokers. Cough was classified as acute in 29 patients, subacute in 114 and chronic in 55. The total duration of cough was 50.3 days on average (17.0 days for acute cough, 36.6 days for subacute cough, and 99.3 days for chronic cough). Out of the patients who complained of cough, $15 \%$ had a history of treatment at other medical institutions. Among those who had been treated at other medical institutions, no one was being treated with inhaled steroids at the time of their visit to our clinic.

The causes of cough in 195 outpatients are listed in Table 1. PIC was the most common etiology of cough in $50.8 \%$ of cases, followed by CVA in $20.5 \%$, and UACS in $14.4 \%$. When the distributions of duration were analyzed, PIC was the cause of acute cough in $75.9 \%$ of patients, subacute cough in $63.2 \%$, and chronic cough in $9.6 \%$. On the other hand, CVA was the second cause of subacute cough in $23.7 \%$ and chronic cough in $25.0 \%$ of cases. Table 2 provides details on differences in characteristics between PIC and CVA. Compared with CVA, patients with PIC had a more recent and shorter duration of cough, shorter treatment period, lower prevalence of having a history of recurrent episode of prolonged cough, higher prevalence of preceding upper respiratory 
Table 2. Clinical characteristics of total patients $(n=195)$

\begin{tabular}{|c|c|c|c|c|}
\hline & $\mathrm{PIC}$ & CVA & Others & $P$-value \\
\hline Number of patients & 99 & 40 & 56 & \\
\hline Age, years & $46.5 \pm 17.6^{*}$ & $51.8 \pm 17.5$ & $53.8 \pm 183$ & 0.04 \\
\hline$\%$ Female & $65.7 \% *$ & $62.5 \%$ & $41.1 \%$ & 0.01 \\
\hline$\%$ Smoker & $18.2 \%$ & $15.0 \% *$ & $32.1 \%$ & 0.08 \\
\hline Duration of cough, days & $32.5 \pm 13.5^{* *}$ & $50.8 \pm 21.1^{* *}$ & $81.9 \pm 79.2$ & $<0.01 * * *$ \\
\hline Duration of cough before the visit, days & $17.8 \pm 10.0^{* *}$ & $23.7 \pm 17.3^{* *}$ & $49.7 \pm 71.6$ & $<0.01$ \\
\hline Period of cough treatment, days & $14.7 \pm 9.0 * *$ & $26.5 \pm 12.5^{*}$ & $32.2 \pm 21.1$ & $<0.01 * * *$ \\
\hline$\%$ Atopy & $37.4 \%$ & $87.5 \% * *$ & $44.6 \%$ & $<0.01 * * *$ \\
\hline$\%$ Recurrent episodes of prolonged cough & $12.1 \% * *$ & $92.5 \%$ & $85.7 \%$ & $<0.01 * * *$ \\
\hline$\%$ Upper respiratory infection & $100.0 \% * *$ & $62.5 \%$ & $64.3 \%$ & $<0.01 * * *$ \\
\hline$\%$ Previous treatment & $16.8 \% * *$ & $11.4 \% * *$ & $22.0 \%$ & $<0.01 * * *$ \\
\hline
\end{tabular}

CVA, cough variant asthma; PIC, post-infection cough. Others include AC, ACE induced cough, CB/COPD, GERD, OSAS and UACS. ${ }^{*} P<0.05$ vs. others, ${ }^{* *} P<0.01$ vs. others, and ${ }^{* * *} P<0.01$ between PIC and CVA. Differences in numerical variables between groups were analyzed by using one-way analysis of variance (ANOVA) and Scheffe's post-hoc test. Differences in categorical variables between groups were statistically tested by using Fisher's exact test, and correction for multiple comparisons was performed using the Benjamini-Hochberg procedure. ${ }^{25}$

infection, and lower atopic predisposition. There was no significant difference between PIC and CVA in the duration of cough before the visit to our clinic.

As AUC is an effective way to summarize the overall diagnostic accuracy of the test, we analyzed AUC to predict PIC and CVA while using the medical history as a clue (Fig. 1). In patients with CVA, having a history of recurrent episode of prolonged cough showed $93 \%$ sensitivity and $60 \%$ specificity [AUC: $0.76,95 \%$ CI (0.71-0.82)] (Fig. 1A). Additionally, the positive likelihood ratio was 2.3 , and the negative likelihood ratio was 0.16 . In patients with PIC, having no history of recurrent episode of prolonged cough showed an $86 \%$ sensitivity and $89 \%$ specificity [AUC: $0.87,95 \%$ CI $(0.82-0.92)$ ] (Fig. 1B). The positive likelihood ratio was 7.8 , and the negative likelihood ratio was 0.16 . In patients with PIC, having a history of "upper respiratory infection" showed a $100 \%$ sensitivity and $37 \%$ specificity [AUC: 0.67 , 95\% CI (0.63-0.73)] (Fig. 1C). The positive likelihood ratio was 1.6 . In patients with CVA, having no history of upper respiratory infection showed a $38 \%$ sensitivity and $86 \%$ specificity [AUC: $0.62,95 \%$ CI $(0.54-0.70)$ ] (Fig. 1D). The positive likelihood ratio was 2.7 , and the negative likelihood ratio was 0.72 .

\section{DISCUSSION}

It is a common consensus in many previous studies that respiratory tract infection and PIC accounts for the highest proportion of acute cough cases ${ }^{10,29-31}$ and is a minor cause of chronic cough. ${ }^{10,12,30,32}$ In our study, PIC was the specified cause of acute cough and chronic cough, occurring with $75.8 \%$ and $8.0 \%$ prevalence, respectively, in patients with cough.

The prevalence of causes of subacute cough depends on the sample size and specialty of the institution. ${ }^{8}, 10,12,31$ Multicenter studies have demonstrated that the prevalence of CVA $(46.9 \%-58.3 \%)$ was higher than that of PIC (11.6\%-12.2\%). ${ }^{10,12}$ On the other hand, Kwon and colleagues demonstrated that the prevalence of CVA (15.8\%) was lower than that of PIC (48.4\%) in single center studies. ${ }^{8}$ Our results, demonstrating a high prevalence of PIC in diseases causing subacute cough, were in concordance with Kwon's study. We speculated that the main reason for this might be that our enrolled patients had no history of treatment with inhaled steroids in other institutions, as this was a similar characteristic in Kwon's patients. In situations where an empirical treatment of PIC did not improve symptoms and CVA was strongly suspected, a steroid treatment was performed. This procedure allowed us to accurately diagnose PIC and CVA.

In primary care clinics, medical history is the most useful tool in clinical reasoning for the diagnosis of diseases. In this study, we showed the utility of some medical histories, such as "recurrent episodes of prolonged cough" and "upper respiratory infection" as a diagnostic approach to identifying the causes of a cough. In patients with asthma, Tomita and colleagues ${ }^{17}$ demonstrated that recurrent episodes of symptoms of cough and/or wheezing is a useful indicator for predicting asthma, with an LR+ of 3.22 and LR- of 1.28. Our study revealed that the recurrent episodes of prolonged 
(A)

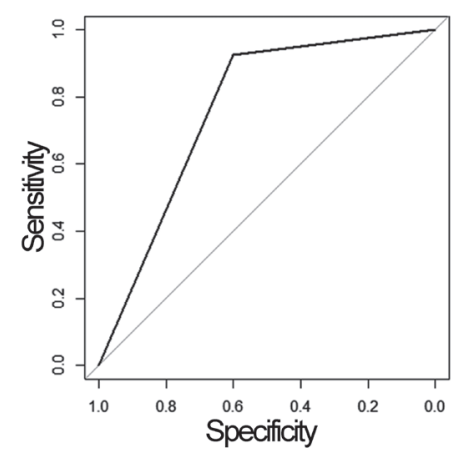

(C)

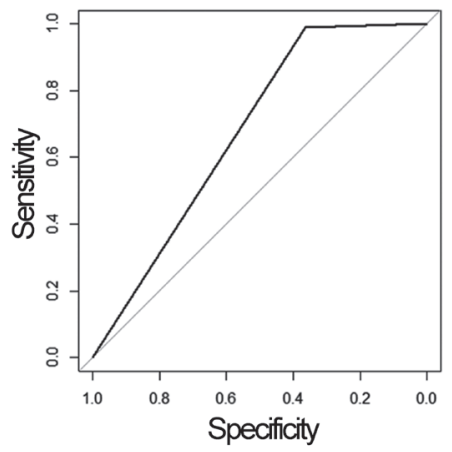

(B)

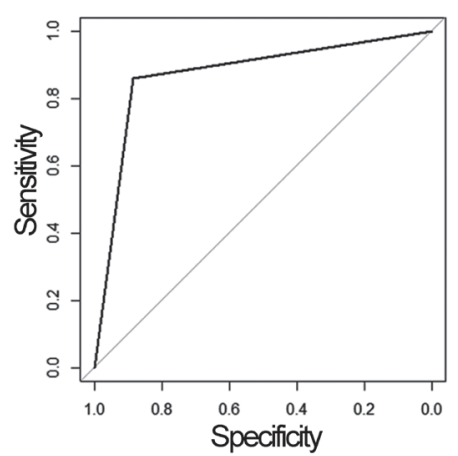

(D)

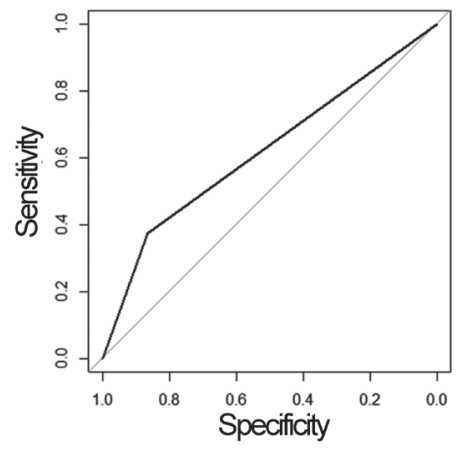

Fig. 1. The ROC curve for a diagnostic test of a patient with a medical history of (A) "recurrent episode of prolonged cough" and its discrimination between CVA and non-CVA; (B) "no recurrent episode of prolonged cough" and its discrimination between PIC and non-PIC; (C) "upper respiratory infection" and its discrimination between PIC and non-PIC; and (D) "no upper respiratory infection" and its discrimination between CVA and non-CVA.

cough noted in the medical history are useful in diagnosing the causative diseases of a cough. Patients with CVA were more likely to have a history of recurrent episodes of prolonged cough (LR+ of 2.3); this result supports Tomita's research. In addition, patients with PIC were more likely to have no history of recurrent episodes of prolonged cough (LR+ of 7.8), which is useful to distinguish PIC from non-PIC.

There are some limitations to this study. First, a relatively small number of patients with cough were examined at a single center. Although the sample size was small, the homogeneity of subjects was preserved, which eliminated any assumption bias. However, a larger study without selection bias is still needed. Another limitation is that we did not assess all potential causes of cough. Some studies reported that chronic cough may have overlapping diseases among asthma, UACS, and GERD, alone or in combination, ${ }^{33}$ as well as overlapping etiologies, including smoking, asthma, and allergic rhinitis. ${ }^{34}$ Since it was reported that eosinophilic airway inflammation is common in subacute cough following acute upper respiratory tract infection, ${ }^{35}$ there is a possibility of overlapping diagnoses with CVA or PIC.

In conclusion, $70 \%$ of causative diseases of cough were CVA or PIC in a primary care setting. Medical histories of "recurrent episodes of prolonged cough" and "upper respiratory infections" were useful clues for predicting CVA and PIC, respectively. However, approximately one-third of patients in primary care with symptoms that are compatible with asthma and normal lung function exhibit airway hyperresponsiveness to methacholine ${ }^{36}$; thus, the limitations of simple spirometry for the diagnosis of asthma must be considered. In the future, the timing of a hospital consultation for cough patients, who were resistant to treatments including ICS, should be studied.

The authors declare no conflict of interest. 


\section{REFERENCES}

1 Smith JA, Woodcock A. Chronic Cough. N Engl J Med. 2017;376:183-4. DOI: 10.1056/NEJMc1615067, PMID: 28076718

2 Finley CR, Chan DS, Garrison S, Korownyk C, Kolber MR, Campbell S, et al. What are the most common conditions in primary care? Systematic review. Can Fam Physician. 2018;64:832-40. PMID: 30429181

3 Irwin RS, Baumann MH, Bolser DC, Boulet LP, Braman SS, Brightling CE, et al. Diagnosis and management of cough executive summary: ACCP evidence-based clinical practice guidelines. Chest. 2006;129(suppl):1S-23S. DOI: 10.1378/ chest.129.1_suppl.1S, PMID: 16428686

4 Morice AH, McGarvey L, Pavord I; British Thoracic Society Cough Guideline Group. Recommendations for the management of cough in adults. Thorax. 2006;61(suppl 1):i1-24. DOI: 10.1136/thx.2006.065144, PMID: 16936230

5 Morice AH, Millqvist E, Bieksiene K, Birring SS, Dicpinigaitis P, Domingo Ribas C, et al. ERS guidelines on the diagnosis and treatment of chronic cough in adults and children. Eur Respir J. 2020;55:1901136. DOI: 10.1183/13993003.01136-2019, PMID: 31515408

6 Kohno S, Ishida T, Uchida Y, Kishimoto H, Sasaki H, Shioya T, et al.; Committee for the Japanese Respiratory Society Guidelines for Management of Cough. The Japanese Respiratory Society guidelines for management of cough. Respirology. 2006;11(suppl 4):S135-86. DOI: 10.1111/j.14401843.2006.00920 1.x, PMID: 16913879

7 Sharma S, Hashmi MF, Alhajjaj MS. Cough. StatPearls Publishing; 2021 Jan. Aug 14. PMID: 29630273

8 Kwon NH, Oh MJ, Min TH, Lee BJ, Choi DC. Causes and clinical features of subacute cough. Chest. 2006;129:1142-7. DOI: 10.1378/chest.129.5.1142, PMID: 16685003

9 Braman SS. Postinfectious Cough. Chest. 2006;129(suppl): 138S-46S. DOI: 10.1378/chest.129.1 suppl.138S, PMID: 16428703

10 Yamasaki A, Hanaki K, Tomita K, Watanabe M, Hasagawa Y, Okazaki R, et al. Cough and asthma diagnosis: physicians' diagnosis and treatment of patients complaining of acute, subacute and chronic cough in rural areas of Japan. Int J Gen Med. 2010;3:101-7. DOI: 10.2147/IJGM.S8167, PMID: 20463827

11 Michaudet C, Malaty J. Chronic cough: evaluation and management. Am Fam Physician. 2017;96:575-80. PMID: 29094873

12 Niimi A, Ohbayashi H, Sagara H, Yamauchi K, Akiyama K, Takahashi K, et al. Cough variant and cough-predominant asthma are major causes of persistent cough: a multicenter study in Japan. J Asthma. 2013;50:932-7. DOI: 10.3109/02770903.2013.823444, PMID: 23841529

13 Dicpinigaitis PV; ACCP Evidence-Based Clinical Practice Guidelines. Chronic cough due to asthma: ACCP evidencebased clinical practice guidelines. Chest. 2006;129(1 suppl):75S-79S. DOI: 10.1378/chest.129.1_suppl.75S, PMID: 16428696

14 Fujimura M, Ogawa H, Nishizawa Y, Nishi K. Comparison of atopic cough with cough variant asthma: is atopic cough a precursor of asthma? Thorax. 2003;58:14-8. DOI: 10.1136/ thorax.58.1.14, PMID: 12511712

15 Matsumoto H, Niimi A, Takemura M, Ueda T, Tabuena R, Yamaguchi $\mathrm{M}$, et al. Prognosis of cough variant asthma: a retrospective analysis. J Asthma. 2006;43:131-5. DOI: 10.1080/02770900500498477, PMID: 16517429
16 Paley L, Zornitzki T, Cohen J, Friedman J, Kozak N, Schattner A. Utility of clinical examination in the diagnosis of emergency department patients admitted to the department of medicine of an academic hospital. Arch Intern Med. 2011;171:1393. DOI: 10.1001/archinternmed.2011.340, PMID: 21824956

17 Tomita K, Sano H, Chiba Y, Sato R, Sano A, Nishiyama O, et al. A scoring algorithm for predicting the presence of adult asthma: a prospective derivation study. Prim Care Respir J. 2013;22:51-8. DOI: 10.4104/pcrj.2013.00005, PMID: 23348712

18 Morice AH, Fontana GA, Sovijarvi ARA, Pistolesi M, Chung KF, Widdicombe J, et al.; ERS Task Force. The diagnosis and management of chronic cough. Eur Respir J. 2004;24:481-92. DOI: 10.1183/09031936.04.00027804, PMID: 15358710

19 Irwin RS, French CL, Chang AB, Altman KW, Adams TM, Altman KW, et al,; CHEST Expert Cough Panel*. Classification of cough as a symptom in adults and management algorithms. Chest. 2018;153:196-209. DOI: 10.1016/ j.chest.2017.10.016, PMID: 29080708

20 Song WJ, Kim HJ, Shim JS, Won HK, Kang SY, Sohn KH, et al. Diagnostic accuracy of fractional exhaled nitric oxide measurement in predicting cough-variant asthma and eosinophilic bronchitis in adults with chronic cough: A systematic review and meta-analysis. J Allergy Clin Immunol. 2017;140:701-9. DOI: 10.1016/j.jaci.2016.11.037, PMID: 28088474

21 Liu W, Jiang HL, Mao B. Chinese herbal medicine for post-infectious cough: A systematic review of randomized controlled trials. Evid Based Complement Alternat Med. 2013;2013:906765. DOI: 10.1155/2013/906765

22 Fokkens WJ, Lund VJ, Mullol J, Bachert C, Alobid I, Baroody F, et al. EPOS 2012: european position paper on rhinosinusitis and nasal polyps 2012. A summary for otorhinolaryngologists. Rhinology journal. 2012;50:1-12. DOI: 10.4193/Rhino50E2, PMID: 22469599

23 El-Serag HB, Sweet S, Winchester CC, Dent J. Update on the epidemiology of gastro-oesophageal reflux disease: a systematic review. Gut. 2014;63:871-80. DOI: 10.1136/ gutjnl-2012-304269, PMID: 23853213

24 The Japanese Respiratory Society. Prolonged and chronic cough. Respirology. 2006;11:S160-74. DOI: 10.1111/j.14401843.2006.00920 8.x

25 Benjamini Y, Hochberg Y. Controlling the false discovery rate: a practical and powerful approach to multiple testing. J R Stat Soc B. 1995;57:289-300. DOI: 10.1111/j.2517-6161.1995. tb02031.x

26 Fagan TJ. Letter: nomogram for Bayes theorem. N Engl J Med. 1975;293:257. DOI: 10.1056/NEJM197507312930513, PMID: 1143310

27 McGee S. Simplifying likelihood ratios. J Gen Intern Med. 2002;17:647-50. DOI: 10.1046/j.1525-1497.2002.10750.x, PMID: 12213147

28 Akobeng AK. Understanding diagnostic tests 3: receiver operating characteristic curves. Acta Paediatr. 2007;96:644-7. DOI: 10.1111/j.1651-2227.2006.00178.x, PMID: 17376185

29 Godycki-Cwirko M, Hood K, Nocun M, Muras M, Goossens $\mathrm{H}$, Butler CC. Presentation, antibiotic management and associated outcome in Polish adults presenting with acute cough/ LRTI. Fam Pract. 2011;28:608-14. DOI: 10.1093/fampra/ cmr019, PMID: 21555340 
30 Fujimura M. Frequency of persistent cough and trends in seeking medical care and treatment-results of an internet survey. Allergol Int. 2012;61:573-81. DOI: 10.2332/allergolint.11OA-0368, PMID: 22918212

31 Otoshi T, Nagano T, Funada Y, Takenaka K, Nakata H, Ohnishi $\mathrm{H}$, et al. A cross-sectional survey of clinical manifestations and underlying illness of cough. In Vivo. 2019;33:5439. DOI: 10.21873/invivo.11508, PMID: 30804139

32 Matsumoto H, Niimi A, Takemura M, Ueda T, Yamaguchi $\mathrm{M}$, Matsuoka $\mathrm{H}$, et al. Prevalence and clinical manifestations of gastro-oesophageal reflux-associated chronic cough in the Japanese population. Cough. 2007;3:1-4. DOI: 10.1186/17459974-3-1, PMID: 17210085

33 Palombini BC, Villanova CAC, Araújo E, Gastal OL, Alt DC, Stolz DP, et al. A pathogenic triad in chronic cough: asthma, postnasal drip syndrome, and gastroesophageal reflux disease. Chest. 1999;116:279-84. DOI: 10.1378/chest.116.2.279, PMID: 10453852
34 Song WJ, Morice AH, Kim MH, Lee SE, Jo EJ, Lee SM, et al. Cough in the elderly population: relationships with multiple comorbidity. PLoS One. 2013;8:e78081. DOI: 10.1371/ journal.pone.0078081, PMID: 24205100

35 Lai K, Lin L, Liu B, Chen R, Tang Y, Luo W, et al. Eosinophilic airway inflammation is common in subacute cough following acute upper respiratory tract infection. Respirology. 2016;21:683-8. DOI: 10.1111/resp.12748, PMID: 26969485

36 Chen A, D’Urzo KA, D’Urzo AD. Airway hyperresponsiveness in patients with normal spirometry results and symptoms compatible with asthma: primary care retrospective chart review. Can Fam Physician. 2021;67:e84-9. PMID: 33727389 